\title{
Pneumatic Launcher Based Precise Placement Model for Large-Scale Deployment in Wireless Sensor Networks
}

\author{
Vikrant Sharma \\ Dept. of Computer Science and Engineering \\ GB Pant Engineering College \\ Pauri, Uttarakhand, India
}

\author{
R B Patel \\ Dept. of Computer Science and Engineering \\ Chandigarh College of Engineering and Technology \\ Chandigarh, India
}

\author{
H S Bhadauria \\ Dept. of Computer Science and Engineering \\ GB Pant Engineering College \\ Pauri, Uttarakhand, India
}

\author{
D Prasad \\ Dept.of Information Technology \\ M M Engineering College \\ Mullana, Haryana, India
}

\begin{abstract}
Sensor nodes (SNs) are small sized, low cost devices used to facilitate automation, remote controlling and monitoring. Wireless sensor network (WSN) is an environment monitoring network formed by the number of SNs connected by a wireless medium. Deployment of SNs is an essential phase in the life of a WSN as all the other performance matrices such as connectivity, life and coverage directly depends on it. Moreover, the task of deployment becomes challenging when the WSN is to be established in a large scale candidate region within a limited time interval in order to deal with emergency conditions. In this paper a model for time efficient and precise placement of SNs in largescale candidate region has been proposed. It constitute of two sets of pneumatic launchers (PLs), one on either side of a deployment helicopter. Each PL is governed by software which determines the launch time and velocity of a SN for its precise placement on the predetermined positions. Simulation results show that the proposed scheme is more time efficient, feasible and cost effective in comparison to the existing state of art models of deployment and can be opted as an effective alternative to deal with emergency conditions.
\end{abstract}

Keywords-WSN; deployment; placement; aerial; coverage

\section{INTRODUCTION}

Sensors are being used over the years to facilitate automation and remote monitoring [1]. Wireless sensors have relieved from the mesh of connecting wires used so far and significantly extended the application domain of sensors. WSNs are widely used for the purpose of disaster management, military, health care, industrial and agricultural monitoring and automation [2][3][4][5]. Deployment is a prime phase in the life of any wireless sensor network (WSN) and the performance of any WSN largely depends on it. It becomes more challenging and difficult when the candidate region is extremely large and unreachable. The matter becomes even more sensitive when it is all about disaster management and life rescue. In such cases a quick, effective and generic technique is required to optimally place the SNs within a candidate region in order to handle the situation.
Deployment can be broadly classified as indoor or open area which is further classified as blanket type, border type and point of interest type [6] [7]. There are scenarios where entire candidate region need to be monitored such as forest fire detection, in such cases SNs are positioned such that complete candidate region is covered and this type of deployment is called blanket type. In many cases particular region need to be isolated from intruders, thus a boundary is made around it by placing the SNs, which detects the movement of intruders, such a deployment is called border type. Even there are cases where only few point within a candidate region need to be monitored such a deployment is called point of interest based deployment.

Various researchers suggested the techniques for the uniform distribution of SNs within a candidate region but none of them considered the emergency conditions raised by natural calamity. In such cases the size of a candidate region is generally large and time is the major constraint to deal with a situation.

In this paper a model for precise and time efficient placement of SNs has been proposed. It is a pneumatic launcher based precise placement model (PLM) which uses a number of SN-launchers powered by the pressure of air. It is a generic model and can be used for the deployment of mobile or static SNs and can be used to effectively deploy SNs for any kind of deployment, such as barrier, blanket or point of interest based.

Rest of the paper is organized as follows. Section II outlines the related work. Preliminary is described in Section III. Section IV constitute of the proposed model. Simulation results are discussed in Section V followed by a conclusion in Section VI.

\section{RELATED WORK}

A lot has been done in the field of deployment of SNs. Initially, Andrew et al. [8] proposed a potential field based 
method of uniform distribution of SNs which assumes that each SNs and obstacles possess a charge due to which they exert a repulsive force on each other. Thus the SNs relocate themselves in order to balance the force exerted by their neighbors thereby distributing themselves uniformly within a candidate region. A Virtual force driven deployment model was proposed by Zou et al. [9], which considers that both repulsive and attractive forces exist between the SNs, If the distance is less than the threshold then there exists a repulsive force if the distance is greater than the threshold then there exists an attractive force. Thus to balance the forces exerted by the neighbors the SNs relocate thereby distributing themselves uniformly within a candidate region. Both the schemes use mobile SNs (MSNs) and focuses on the uniform distribution of the SNs but least concern was given to the connectivity with the base station (BS). Connectivity Preserved virtual force (CPVF) and FLOOR based schemes were proposed by Guang et al. [10] to deal with the BS connectivity issues.

Corke et al. [11][12] proposed deployment model which uses a robot helicopter equipped with screw groove assembly to carry and precisely drop the SNs to predefined locations. Although, the model precisely deploys the SNs within a candidate region, it is not feasible to be used for large scale deployments, due to limited battery life and carrying capacity of the robot helicopter. Yoshiaki et al.[13], proposed a uniform aerial deployment (UAD) model to deploy SNs from air in large scale candidate region. It uses special parachute to carry a SN. The Parachutes are assumed to have a capability to switch between "gliding" and "falling" states in order to achieve the required density level. Although, UAD is an improved mechanism for aerial scattering, but it can only work for the SNs falling at the same level (altitude) and the design of a parachute is also not defined.

In [14] authors proposed a Centrifugal Cannon based Sprinkler (CCS) to randomly scatter the SNs within a candidate region. CCS is an assembly of variable sized cannons rotated by a motor at specified RPM. It alone cannot yield the coverage equivalent to optimal, as it is a random scattering model, but it provides an effective and time efficient method for random scattering of SNs over large unreachable regions.

In order to achieve the blanket coverage over a large-scale candidate region, most of the previously proposed state of art models either randomly scatters the SNs from the air or use MSNs, which are programmed to relocate to the optimal locations after random dropping. While, other uses robot helicopter to precisely place the SNs on the pre-computed, optimal locations within a candidate region. Among these models, although the random scattering model of $\mathrm{SN}$ deployment is the simplest, but it cannot yield optimal coverage. Usage of MSNs In place of static SNs emerged as an effective solution for the optimal deployment problem of randomly scattered SNs but, MSNs are relatively costlier and have their own limitations of mobility in uneven and diverse terrain.

\section{PRELIMINARY}

\section{A. Deployment helicopter}

It traverses the entire candidate region in order to aerially deploy the SNs.

\section{B. Deployment path}

It is a virtual track on which the deployment helicopter moves while traversing the candidate region.

\section{Path width}

It is width of a strip on the candidate region covered by ECCS. It is equal to the twice of the horizontal distance covered by a SN launched from the longest cannon.

\section{Base line}

It is a virtual path above which the deployment helicopter moves, while traversing the candidate region.

\section{E. Optimal deployment}

Motivated from the cellular architecture of mobile networks, the entire candidate region is divided into hexagonal cells and center of these cells forms the optimal deployment locations (shown in Fig. 1).

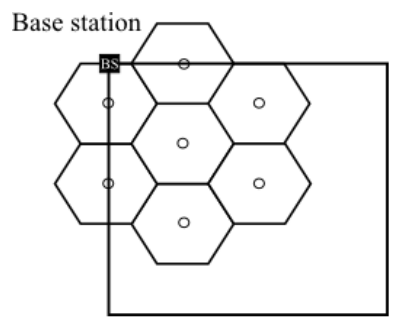

Fig. 1. Optimal deployment pattern

\section{PROPOSED MODEL}

\section{A. Model Assumptions}

It is assumed that SNs are encapsulated within a spherical casing, in order to ensure the evenness in shape and to protect them from any kind of physical damage while landing. The deployment helicopter is equipped with precise positioning system. The density of air $\rho$ is assumed to be constant, i.e., $1.255 \mathrm{Kg} / \mathrm{m}^{3}$. Mass, M of SNs is $0.250 \mathrm{Kg}$.

Prior information such as buildings, water bodies or any other structure where deployment is not required is available either in the form of digital map or satellite image. 


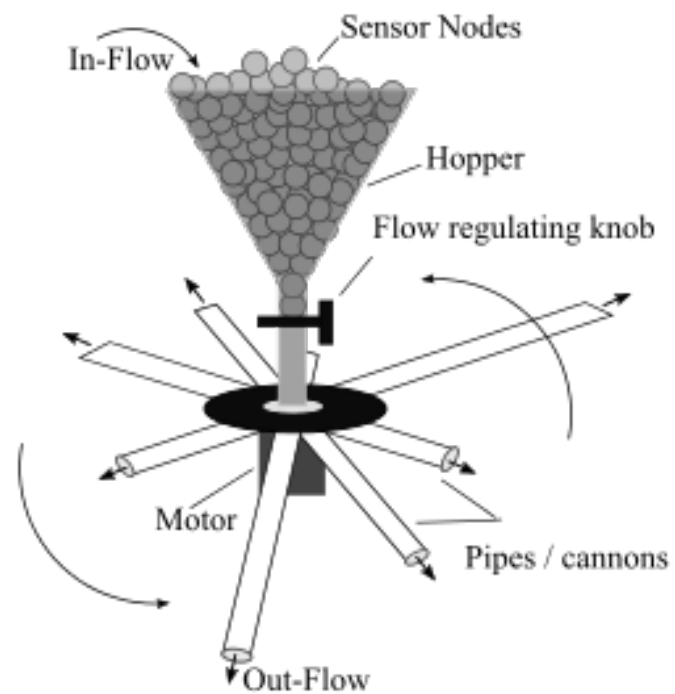

Fig. 2. Centrifugal cannon based sprinkler (CCS)

\section{B. Centrifugal Cannon based Sprinkler (CCS)}

CCS was designed as a time efficient and effective method to randomly scatter the SNs within a large scale candidate region. It constitute of assembly of variable sized cannons rotated by a motor to sprinkle the SNs within a candidate region (see Fig. 2). CCS is mounted on a deployment helicopter, which traverses the entire candidate region while following a predefined scan-path.

\section{Pneumatic launcher based model (PLM)}

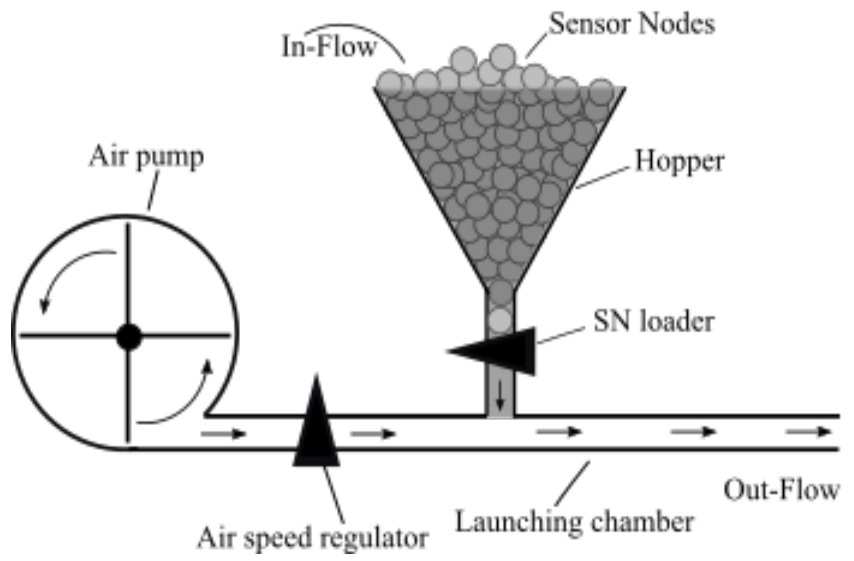

Fig. 3. Pneumatic launcher

Following are the prime components of a pneumatic launcher:

- Hopper: SNs are collectively held inside the hopper, from where they are sequentially loaded into the launching chamber.

- Air pump: It is a centrifugal pump, driven by a high speed DC motor. It blows the air through the launching chamber.

- Launching chamber: It is a pipe, connected to the hopper and air pump as shown in Fig. 3. It launched the SNs with the thrust of air pumped by the air pump.
- Air speed regulator: It controls the flow of air through the launching chamber, so as to ascertain the required launch velocity of a SN.

- SN loader: Loads the SN into the launching chamber.

- SN capsule: The SN is placed within a spherical shell, so as to ensure the evenness and alike shape of each $\mathrm{SN}$. It is made up of two concentric spheres. The inner part is made up of shock absorbing material (e.g. sponge or thermocol) in order to cushion the $\mathrm{SN}$ and absorb the shocks generated while landing. The outer part is made up of a thin layer of hard and brittle material. The capsule is divided into two hemispheres (i.e., upper and lower), both containing a groove to pack a SN within. Bottom of a lower-half is filled with a sticky gel, in order to keep the bottom heavy for ensuring the landing position, absorb the landing shock and minimize the post landing movements of SN.

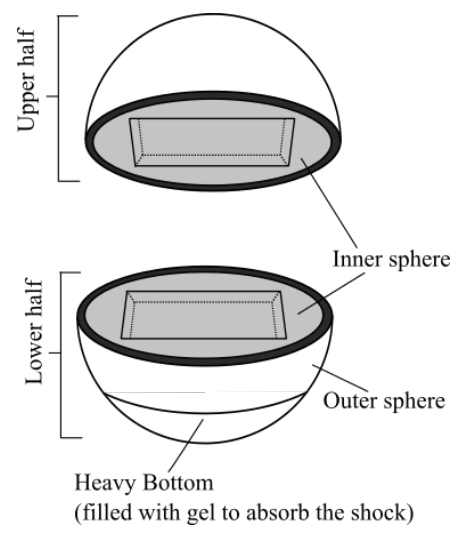

Fig. 4. SN capsule

1) Pre-deployment configuration and computation: The Entire candidate region is divided into hexagonal cells (regular hexagons with each side equal to $r_{s}$ ) to achieve the optimal coverage and center of these hexagons form the DLs for the placement of SNs. The cellular division of candidate region is motivated from cellular networks [15]. The relation between $r_{c}$ and $r_{s}$ is given by equation (1).

$$
r_{c}=\sqrt{3} * r_{s}
$$

The helicopter follows a pre-defined path in order to traverse the entire candidate region. The candidate region is marked by the master grid consisting of vertical and horizontal lines formed by joining the adjacent DLs as shown in Fig 5 .

The horizontal lines are labeled as:

$H B_{-n}^{0}, H B_{-n+1}^{0}, H B_{-n+2}^{0}, \ldots, H B_{0}^{0}, H B_{1}^{0}, H B_{2}^{0}, \ldots, H B_{n}^{0}$, $H B_{-n}^{1}, H B_{-n+1}^{1}, H B_{-n+2}^{1}, \ldots, H B_{0}^{1}, H B_{1}^{1}, H B_{2}^{1}, \ldots, H B_{n}^{1}$, $H B_{-n}^{k}, H B_{-n+1}^{k}, H B_{-n+2}^{k}, \ldots, H B_{0}^{k}, H B_{1}^{k}, H B_{2}^{k}, \ldots, H B_{n}^{k}$, where $n$ is the number of PLs in each set of PLM and $k$ is the total number of parallel scan lines. The distance between adjacent horizontal lines $d_{h}$ and adjacent parallel scan path $d_{p}$ is given by equation (2) and (3) respectively. However, the total number of parallel-line scan paths is given by equation (4). 


$$
\begin{gathered}
d_{h}=\frac{3 r_{s}}{2} \\
d_{p}=2 * n * \frac{3 r_{s}}{2} \\
k=\frac{w}{d s}
\end{gathered}
$$

where $w$ is the width of a candidate region.

The helicopter moves above the parallel-line scan path labeled as $H B_{0}^{X}$. Vertical lines are labeled as $B_{1}, B_{2}, \ldots, B_{m}$, where $m$ is the total number of vertical lines and its value depends on the width of a candidate region. The distance $d_{v}$ between adjacent vertical lines is given by equation (5).

$$
d_{v}=\frac{\sqrt{3} r_{s}}{2}
$$

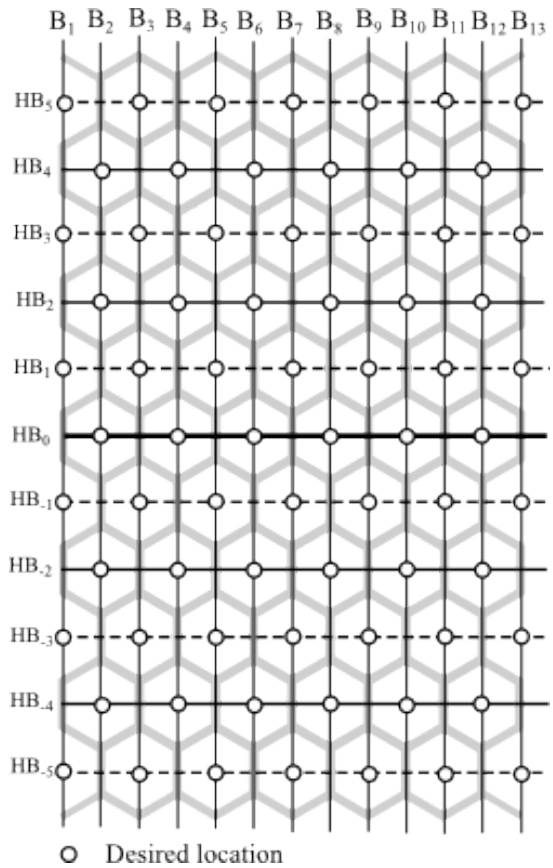

Fig. 5. Logical division of a candidate region

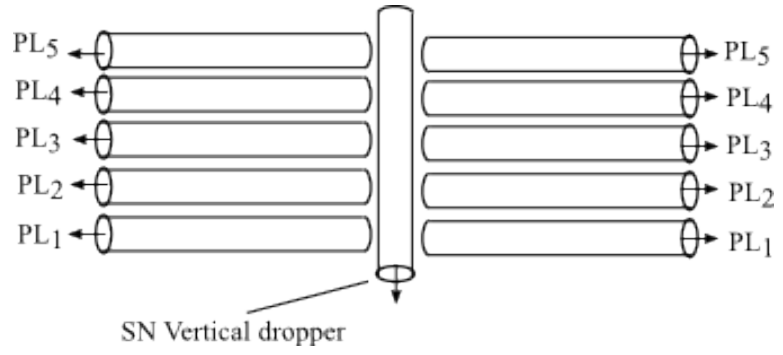

Fig. 6. Sets of PLs used in PLM

PLM consist of two sets of pneumatic launchers, $P L=$ $\left\{P L_{1}, P L_{2}, P L_{3}, \ldots, P L_{n}\right\}$, one on each side of a helicopter, all the pneumatic launchers are of equal lengths $l$ but have variable range of launch velocities. Launch velocities $V L_{i}$ of any pneumatic launcher $P L_{i}$ is given by the corresponding elements set $V L$.

$V L=\left\{V L_{1}, V L_{2}, V L_{3}, \ldots, V L_{n}\right\}$, such that, $V L_{1}<V L_{2}<$ $V L_{3}<\ldots<V L_{n}$.

The computation of value of $V L_{i}$ is further discussed in this section.

2) Without air resistance: The time of flight, $t$ of $\mathrm{SN}$ fired from $P L_{i}$ is given by equation (2).

$$
t=\sqrt{\frac{2 H}{g}}
$$

Where, $H$ is a dropping height and $\mathrm{g}$ is the acceleration due to gravity $(\mathrm{g}=9.8)$. Horizontal distance $D_{h}$ covered by SN fired from $P L_{i}$ is given by equation (3)

$$
D_{h}=V L_{i} * \sqrt{\frac{2 H}{g}}
$$

3) With air resistance: In real scenarios, the air plays an important role in determining the trajectory of a launched SN. The launched SN stops accelerating vertically after achieving its terminal velocity $v_{t}$, due to the resistance offered by the air. Terminal velocity is a function of weight, radius of spherical $\mathrm{SN}$ and density of air (given in equation (8)).The relation between weight, radius and terminal velocity of a spherical SN is shown in shown in Fig. 7.

$$
v_{t}=\sqrt{\frac{2 M g}{C_{d} \rho A}}
$$

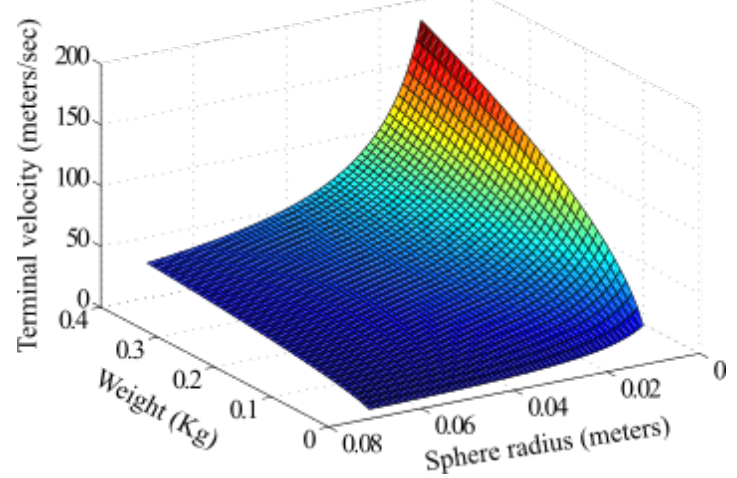

Fig. 7. Relation between radius, weight and terminal velocity of SN

Resistive force $F_{d}$ is exerted by air on every SN fired from PLM, which reduces the horizontal distance covered by it. The value of $F_{d}$ is given by equation (9).

$$
F_{d}=\frac{1}{2} v^{2} \rho A C_{d}
$$

Where, $v$ is the current speed of $\mathrm{SN}, A$ is the area of crosssection of SN's capsule, $\rho$ is the density of air (i.e., 1.255 $\mathrm{Kg} / \mathrm{m}^{3}$ ) and $C_{d}$ is a drag-coefficient for sphere (0.5). Fig. 8 (a) and (b) represents the trajectory formed by the SNs fired from PLM (with and without air resistance, respectively). 


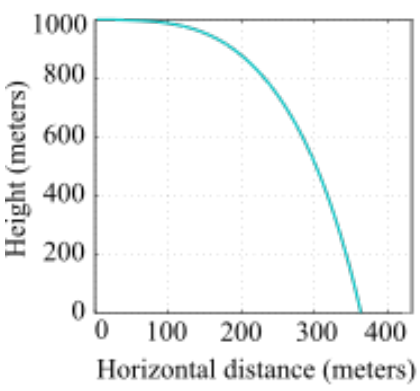

(a)

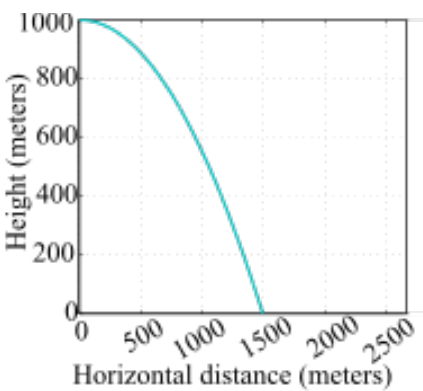

(b)
Fig. 8. (a). Trajectory formed by SN fired from PLM (With air). (b). Trajectory formed by SN fired from PLM (Without air)

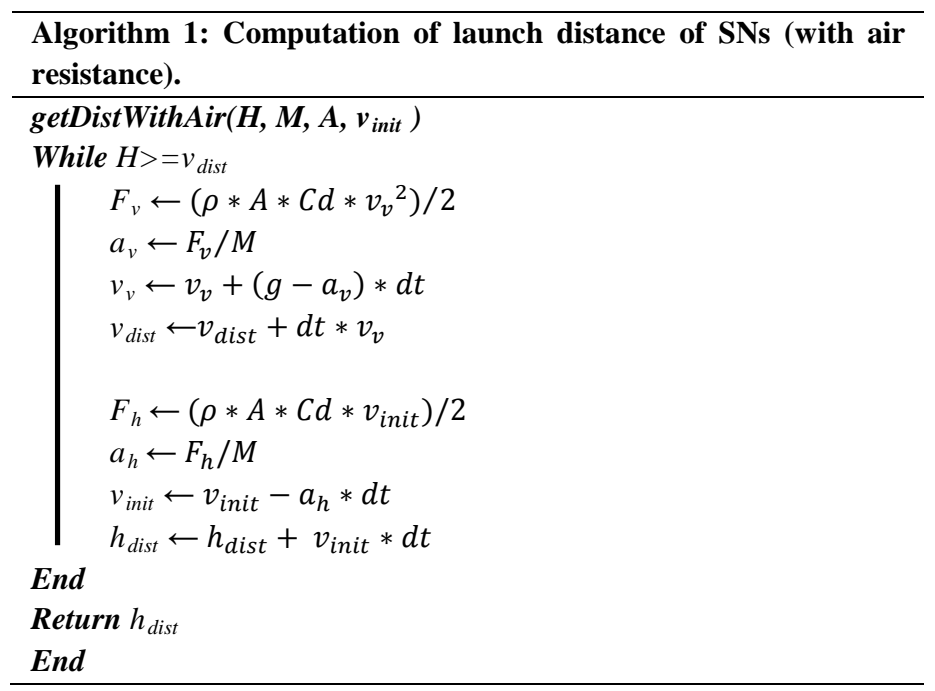

4) Computation of launch velocity: Launch velocity is a speed with which the SN is launched from the $P L$. Each $P L$ in a set is assigned different magnitude of launch velocity. It is adjusted such that the projectile formed by the launch from each $P L$ ends on the corresponding $H B$.

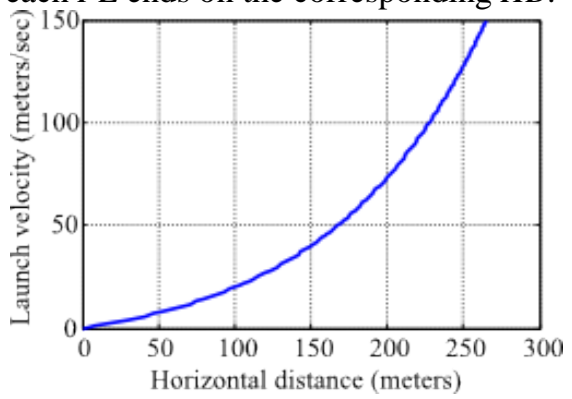

Fig. 9. Relation between VL and horizontal distance covered

Graph in Fig. 9 represents the relation between $V L$ and horizontal distance covered by a SN. The deployment height considered to be $200 \mathrm{~m}$. $V L_{i}$ for particular $P L_{i}$ is adjusted such that the SN launched by $P L_{i}$ covers the horizontal distance $D_{i}$ before hitting the ground. $D_{i}$ is the distance between baseline and the horizontal line $H B_{i}$.

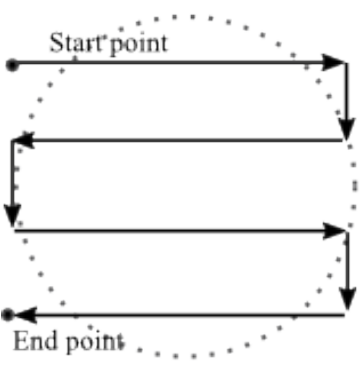

(a)

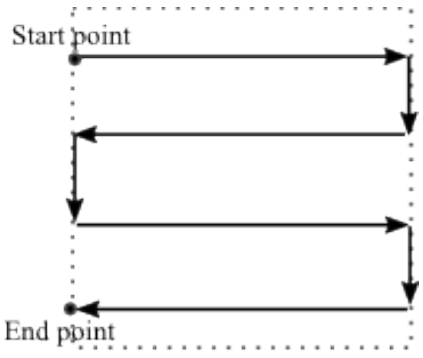

(b)
Fig. 10. (a) Scan path for circular candidate region. (b) Scan path for rectangular candidate region

5) Scan Path: It is a path defined for a deployment helicopter to traverse the candidate region while deploying the SNs [14]. In this paper we have considered the scan path in the form of horizontal parallel lines (see Fig. 10). SNs are precisely launched by the PLM while moving in the scan path. Operation of PLM is given by Algorithm 2.

6) $S N$ horizontal-launch regulation: Launch regulation is an important task performed by PLM. It depends on the length of the $P L_{i}$ and its $V L_{i}$. Each loaded $S N$ passes through the $P L_{i}$ in time interval

Each $P L_{i}$ consumes a specific time $T_{i}$ to launch a $\mathrm{SN}$, called dispense time (given in equation (10)).

$$
T_{i}=T_{L}+t_{i}
$$

where $T_{L}$ is the time taken by the actuator to load the SN into the cannon and $t_{i}$ (given in equation (11)) is the time taken by the SN to pass through $P L_{i}$.

$$
t_{i}=\frac{2 l}{V L_{i}}
$$

Vertical lines are categorized as real-vertical lines $\left(R B_{j}\right)$ and virtual-vertical lines $\left(V B_{j}\right) . R B_{j}$ constitutes of actual lines which are plotted on the ground on the basis of computed DLs, while the $V B_{j}$ is the copy of $R B_{j}$ above the ground at height $H$, with a shift of distance $D_{s}$. The shift is opposite to the direction of movement of the helicopter as shown in Fig. 11 and its value is given by equation (12). It is done in order to compensate the displacement caused due inertia induced in the SN by the movement of the helicopter.

$$
D_{s}=\text { getDistWithAir }\left(H, M, A, V_{H}\right)
$$

where $H$ is the altitude of a deployment helicopter, $\mathrm{M}$ is the mass of SNs, $A$ is the cross-sectional area of the SN and $V_{H}$ is the velocity with which the helicopter.

The errors are introduced while deployment of SNs due to various unavoidable factors such as environmental winds, humidity and temperature. These errors are called uncertainty errors $E_{u}$ and their magnitude largely depends on the height of deployment. In this paper the value of $E_{u}$ is considered as $7.5 \%$ of $\mathrm{H}$. 


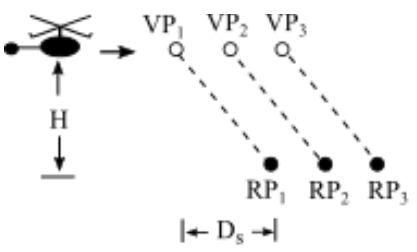

(a)

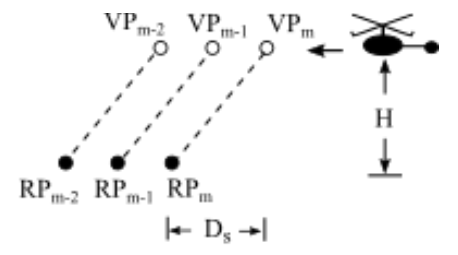

(b)
Fig. 11. (a). Vertical line-shift on left to right movement of deployment helicopter. (b). Vertical line-shift on right to left movement of deployment helicopter

7) Exception handling: Exceptions are the selected regions within a candidate region where deployment is not required these may include water bodies, buildings or any other structure or selected region. The DLs overlapping these regions are located on the digital map of a candidate region and removed from the set of DLs before feeding DLs to the PLM.

\section{Algorithm 2: PLM operation}

- moveOnPath(speed, altitude): Moves the deployment helicopter above the scan path $\left(\mathrm{HP}_{0}^{\mathrm{X}}\right)$ at specific altitude with specific speed.

- getNextVPOnPath(): Returns the next vertical line $\left(\mathrm{VP}_{\mathrm{X}}\right)$ on the scan path.

- getVPNumber(): Returns the reference number of a vertical line.

- $\quad \operatorname{dropSN():~Simply~drops~the~SN~vertically~without~using~}$ the cannons.

- loadEvenCannons(): Loads cannons labeled with even number.

- loadOddCannons(): Loads cannons labeled with even number.

- currentX: X-coordinate at current position of deployment helicopter.

\section{Thread 1}

1. $\quad$ startMovingOnPath(speed, altitude);

2. $\operatorname{setRPM(rpm);}$

\section{Thread 2}

Till endOfPath do

\section{If $V P !=$ getNextVPOnPath() Then}

$V P \leftarrow$ getNextVPOnPath(); If $\mid$ current $X-V P . X \mid=$ speed $* T_{i}$ Then If $V P$.get $V P N u m b e r \% 2=0$ Then loadEvenCannons(VP); dropSNonPath();

Else IoadOddCannons();
End End

End

End

\section{dropSNonPath(VP)}

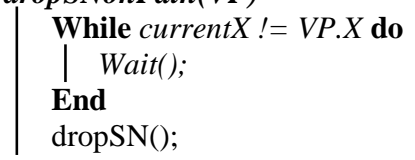

\section{Simulation Results AND Discussion}

The simulation of the proposed model has been performed Quorum Comm (our own simulator developed in java). Simulation is repeated 500 times and the average values are presented as the results. The values of variables used while simulation is given in TABLE I. Since this model is a unique of its kind, not much is available for comparison

Fig. 12 represents the coverage pattern of PLM as well as CCS. It is observed that the coverage achieved by PLM is very close to that of optimal. It uniformly covers the maximum part of a candidate region (see Fig. 12 (a)). However the coverage pattern achieved by CCS comparatively non-uniform (see Fig. 12 (b)).

TABLE I. SIMULATION PARAMETERS

\begin{tabular}{ll}
\hline Parameter & V \\
Communication range $\left(r_{c}\right)$ & 70 \\
Sensing range $\left(r_{s}\right)$ & \\
Sprinkler RPM & \\
Height of deployment $(H)$ & \\
SN radius & \\
SN weight & \\
Helicopter speed $\left(V_{H}\right)$ & \\
Uncertainty error $\left(e_{u}\right)$ & \\
Area of candidate region \\
Number of SNs \\
Number of PLs in a set \\
\\
Desired Location
\end{tabular}

(a)

Value
$70 \mathrm{~m}$
$40 \mathrm{~m}$
1000
$200 \mathrm{~m}$
$0.05 \mathrm{~m}$
$0.25 \mathrm{Kg}$
$27.7 \mathrm{~m} / \mathrm{s}(100 \mathrm{Km} / \mathrm{h})$
$7.5 \%$ of $H$
$1000 \mathrm{~m} \mathrm{X} 1000 \mathrm{~m}$
250
5

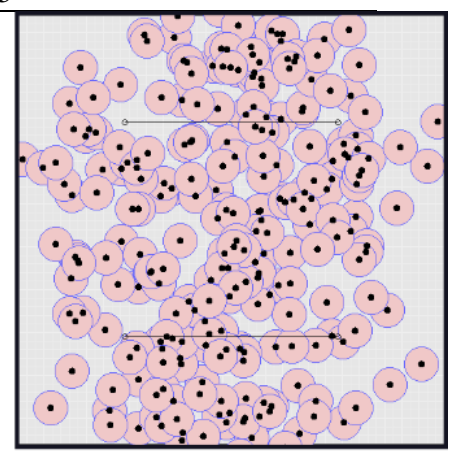

(b)

Fig. 12. (a) Coverage by PLM (b) Coverage by CCS

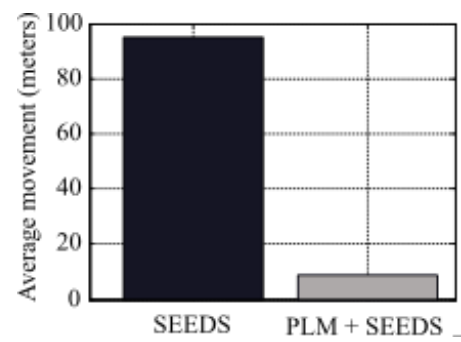

Fig. 13. Enhanced perfomance of SEEDS with PLM

Fig. 13 demonstrates the impact of PLM on SEEDS. SEEDS is a relocation based deployment scheme which uses MSNs to facilitate mobility. The average movement of MSNs in SEEDS is reduced from $95 \mathrm{~m}$ to $9.5 \mathrm{~m}$ when PLM is used to scatter the MSNs. 


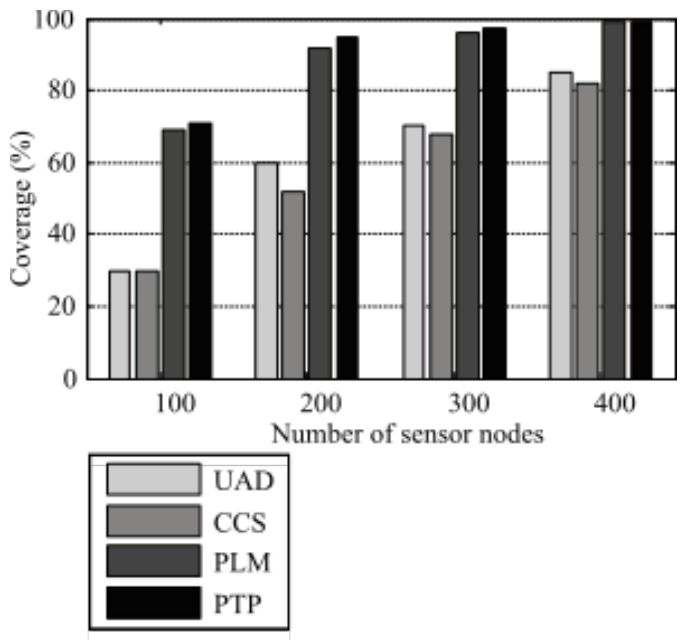

Fig. 14. Coverage achieved by various deployment models

The coverage achieved by PLM is very close to the optimal. Comparison of coverage achieved by various aerial deployment schemes is shown in Fig. 14.

Fig. 15 represents the comparison between the time taken by PTP and PLM to deploy the SNs. It is observed that PLM is 5.2 (approx) times faster than PTP deployment model and yields approximately same coverage. It is due to the fact that PLM covers the wide band on the candidate region in a single scan, thus minimizing the number of scans to deploy the SNs.

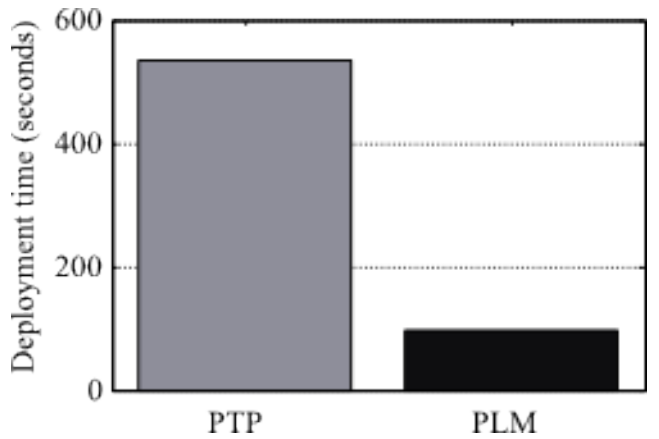

Fig. 15. Comparison between deployment time taken by PTP and PLM

\section{CONCLUSION}

In this paper a model for time efficient and precise placement of SNs in large-scale candidate region has been proposed. It constitute of two sets of PLs, one on either side of a deployment helicopter. SNs are launched from these PLs with controlled velocity and time such that they land on the pre-computed locations. Each PL is governed by software which determines the launch time and velocity of a SN for its precise placement. Simulation results show that the proposed scheme is more time efficient, feasible and cost effective in comparison to the existing state of art models of deployment. We are designing a hardware model of PLM for its hardware level testing and implementation. Moreover, the future enhancements of PLM include its generalization for all types of terrain.

\section{REFERENCES}

[1] Kun Yang, "Wireless sensor networks," Principles, Design and Applications, 2014.

[2] Mainwaring Alan, Polastre Joseph, Szewczyk Robert, and Culler David, "Wireless sensor networks for habitat monitoring," in WSNA '02 Proceedings of the 1st ACM international workshop on Wireless sensor networks and applications, New York, 2002, pp. 88-97.

[3] G W Allen et al., "Deploying a Wireless Sensor Network on an Active Volcano," IEEE Internet Computing, vol. 10, no. 2, pp. 18-25, April 2006.

[4] M Felamban, B Shihada, and K Jamshaid, "Optimal node placement in underwater wireless sensor networks," in 27th International Conference on Advanced Information Networking and Applications (AINA), 2013 IEEE, 2013, pp. 492-499.

[5] Zhi Sun et al., "BorderSense: Border patrol through advanced wireless sensor networks," Ad Hoc Networks, vol. 9, no. 3, pp. 468-477, May 2011.

[6] Vikrant Sharma, RB Patel, HS Bhadauria, and D Prasad, "Deployment schemes in wireless sensor network to achieve blanket coverage in large-scale open area: A review," Egyptian Informatics Journal, p. in press, 2015.

[7] Shigeaki Tanabe, Kei Sawai, and Tsuyoshi Suzuki, "Sensor node deployment strategy for maintaining wireless sensor network communication connectivity," International Journal of Advanced Computer Sciences and Applications, vol. 2, no. 12, pp. 140--146, 2011.

[8] Andrew Howard, Maja J Matari'c, and Gaurav S Sukhatme, "An incremental self-deployment algorithm for mobile sensor networks," Autonomous Robots, vol. 13, no. 2, pp. 113-126, 2002.

[9] Yi Zou and Krishnendu Chakrabarty, "Sensor deployment and target localization based on virtual forces," in INFOCOM 2003. TwentySecond Annual Joint Conference of the IEEE Computer and Communications. IEEE Societies, San Francisco, 2003, pp. 1293-1303.

[10] Guang Tan, Stephen A Jarvis, and A-M Kermarrec, "Connectivityguaranteed and obstacle-adaptive deployment schemes for mobile sensor networks," IEEE Transactions on Mobile Computing, vol. 8, no. 6, pp. 836-848, 2009.

[11] P. Corke, S. Hrabar, R. Peterson, D. and Saripalli, S. Rus, and G. Sukhatme, "Autonomous deployment and repair of a sensor network using an unmanned aerial vehicle," in IEEE International Conference on Robotics and Automation, 2004, pp. 3602-3608.

[12] Peter Corke et al., "Deployment and connectivity repair of a sensor net with a flying robot," Experimental robotics IX, pp. 333-343, 2006.

[13] Yoshiaki Taniguchi, Tomoya Kitani, and Kenji Leibnitz, "A uniform airdrop deployment method for large-scale wireless sensor networks," International Journal of Sensor Networks, Inderscience, vol. 9, no. 3/4, pp. 182-191, 2011.

[14] V Sharma, R B Patel, H S Bhaduria, and D Prasad, "Policy for random aerial deployment in large scale Wireless Sensor Networks.," in International Conference on Computing, Communication Automation (ICCCA), 2015, Noida, 2015, pp. 367-373.

[15] Stuber and L Gordon, Principles of mobile communication, 3rd ed. Atlanta, USA: Springer, 2011. 